\title{
A Practical Method for Peach-related Species Identification and Hybrid Analysis Using Simple Sequence Repeat Markers
}

\author{
Zuguo Cai \\ Zhengzhou Fruit Research Institute, Chinese Academy of Agricultural Sciences, Zhengzhou 450009, \\ People's Republic of China; and College of Horticulture and Landscape Architecture, Henan Institute \\ of Science and Technology, Xinxiang 453003, People's Republic of China
Wenfang Zeng, Liang Niu, Zhenhua Lu, Guochao Cui, Yunqin Zhu, Lei Pan, Yifeng Ding, and Zhiqiang Wang ${ }^{1}$
Zhengzhou Fruit Research Institute, Chinese Academy of Agricultural Sciences, Zhengzhou 450009, People's Republic of China

\begin{abstract}
AdDITIONAL INDEX wORDS. microsatellite marker, DNA fingerprinting, SSR, Prunus persica, National Germplasm Repository of Peach in China, NGRPC

Abstract. Cultivated peach (Prunus persica) is an important fruit species worldwide. The wild relatives in Prunus, such as $P$. mira, $P$. davidiana, $P$. kansuensis, $P$. ferganensis, and $P$. persica, are valuable for peach breeding, and early and accurate identification of parental and hybrid genotypes is critical. In this study, 20 representative accessions of peach germplasm from the National Germplasm Repository of Peach in China were used to select a set of 18 simple sequence repeat (SSR) markers for accurate species discrimination. Eight unknown peach samples were successfully identified using the SSR panel and species genotype database. Interspecific hybrid genotypes of $P$. persica $\times P$. davidiana, $P$. persica $\times P$. kansuensis, and $P$. persica $\times P$. ferganensis were also analyzed reliably. The markers were amenable to high-throughput fluorescent labeling and capillary electrophoresis (CE) analysis, allowing rapid and efficient species identification. The practical method described in this study will facilitate peach breeding and germplasm management.
\end{abstract}

Peach is one of the most important fruit species worldwide. China, where peach originated, has the richest resource of species related to cultivated peach. These species contributed to peach domestication history over more than 4000 years, with some cultivars disseminating to Central Asia, the Mediterranean coast, Europe, America, and Japan (Layne and Bassi, 2008; Wang and Zhuang, 2001). The main relatives of cultivated peach, namely $P$. mira, $P$. davidiana, $P$. kansuensis, and $P$. ferganensis, are found readily in southwestern and northwestern China. Fruit from almost all of the related wild species exhibit poor eating quality, but these species could be valuable as sources of pest and disease resistance traits or as rootstock (Cao et al., 2011; Moing et al., 2003). Increasing numbers of related species are being used to develop new peach cultivars. Efficient and accurate species identification and hybrid analysis are necessary in peach breeding and germplasm management.

A range of practices were used previously to identify peachrelated germplasm, including morphology (Wang and Zhuang, 2001), cytology (Guo et al., 1996), palynology (Wang and Zhou, 1990), isozymes (Mowrey et al., 1990), and DNA markers (Cheng et al., 2001; Yu et al., 2004). With the development of sequencing technology, peach genome sequences have become available (Verde et al., 2013), and high-throughput single-

Received for publication 23 Sept. 2016. Accepted for publication 2 Feb. 2017. The research was financially supported by the Agricultural Science and Technology Innovation Program (ASTIP) (CAAS-ASTIP-2017-ZFRI), the National Key Technology Support Program of China (2013BAD02B03-2; 2014BAD16B04).

We thank Gengrui Zhu, Mingliang Yu, and Quan Jiang providing peach-related species samples.

${ }^{1}$ Corresponding author. E-mail: wangzhiqiang@caas.cn. nucleotide polymorphism (SNP) tools have been used for largescale genetic analysis in peach cultivars and accessions (Cao et al., 2014; Verde et al., 2012).

SSR (microsatellites) are tandem repeat DNA sequences with a core unit of 1-6 bps, which are abundant in prokaryotic and eukaryotic genomes and are ubiquitously distributed in both the protein-coding and noncoding regions (Guichoux et al., 2011; Kalia et al., 2011). The high variability of microsatellites usually manifests as different numbers of repeats in the region of the repeated motif, and short insertion/deletion events are also seen (Decroocq et al., 2003). SSR markers are ideal for profiling as they are codominant and can display a large number of alleles per locus. In addition, assaying SSR markers is relatively simple and reproducible and is accomplished using polymerase chain reaction (PCR)-based methods that are amenable to automation (Kalia et al., 2011). SSR markers proved to be highly efficient for genetic analysis in several fruit species, such as grape [Vitis vinifera (Emanuelli et al., 2013)], jujube [Ziziphus jujuba (Ma et al., 2012)], almond [Prunus dulcis (Dangl et al., 2009)], and sweet cherry [Prunus avium (Lacis et al., 2009)]. SSR markers were developed previously in peach and used for genetic diversity assessment (Li et al., 2008), cultivar identification (Chen et al., 2011; Li et al., 2013), trait mapping (Lambert and Pascal, 2011; Liu et al., 2009), and phylogenetic studies (Cheng and Huang, 2009). However, to date, reliable and efficient SSRs for discrimination of peach-related species have not been developed, and no database of species genotypes has been established. A universal database of SSR marker profiles from peach-related species would facilitate peach breeding and germplasm management.

The NGRPC, located in Zhengzhou (central China), Nanjing (eastern China), and Beijing (northern China), is the most 
important germplasm repository of peach, nectarine, and related species in China. The NGRPC preserves original and representative accessions of species related to peach and is the core collection of such species for China and the world. Here, we describe a practical SSR-based method for discriminating species related to peach and for identifying interspecific hybrids. The aims of this study were to 1) screen SSRs and develop a set of markers for efficient identification of peachrelated species, 2) establish a universal genotype database for peach-related species using fluorescent-labeled SSR markers, and 3) demonstrate the practical utility of the SSR set for unknown genotype identification and interspecific hybrid analysis.

\section{Materials and Methods}

Plant material. Twenty-nine Prunus samples were used in the study (Table 1). Among them, 20 samples (codes 1-20) were obtained from three NGRPCs (Zhengzhou, Nanjing, and Beijing). These 20 accessions were representative samples of five species $(P$. persica, $P$. davidiana, $P$. kansuensis, $P$. ferganensis, and $P$. mira) that were established on the core collection during initial investigations in China in the last century. Some of these representative accessions were described using classical morphological characteristics, regional origin, and landrace names. Eight samples (codes 21-28) were unknown accessions awaiting species identification, and one sample (code 29) was a cultivar that was used as a maternal parent for hybridization. Three interspecific hybridizations were performed by the Zhengzhou Fruit Research Institute, Chinese Academy of Agricultural Sciences (ZFRI, CAAS), as follows: $P$. persica (code 29) $\times P$. davidiana (code 1), $P$. persica $($ code 29$) \times P$. kansuensis (code 5), and $P$. persica (code 29) $\times P$. ferganensis (code 10 ). Three $\mathrm{F}_{1}$ hybrid populations were obtained and used alongside their parents for hybrid analysis.

DNA extraction. Fresh young leaves were collected and rapidly dried at room temperature using solid silica gel pellet desiccant. Genomic DNA was isolated using the modified cetyltrimethylammonium bromide protocol described by Cheng et al. (1997). DNA was quantified using a spectrophotometer (BioPhotometer plus; Eppendorf AG, Hamburg, Germany), and diluted to $20 \mathrm{ng} \cdot \mu^{-1}$ as template for PCR amplification.

SSR MARKERS. Seventy-eight SSR markers (Table 2), previously developed for peach (Aranzana et al., 2002; Dirlewanger et al., 2002; Sosinski et al., 2000; Testolin et al., 2000; Yamamoto et al., 2002), japanese plum [Prunus salicina (Mnejja et al., 2004)], and almond (Mnejja et al., 2005), were tested for their ability to produce amplification products in Prunus species. The 78 loci were distributed across the whole Prunus genome, with 9-10 loci per linkage group.

PCR AMPlifiCATION. PCR amplification was performed in $20-\mu \mathrm{L}$ reaction volumes containing $20 \mathrm{ng}$ of genomic DNA, 10 $\mu \mathrm{L}$ of $2 \times$ Taq PCR MasterMix (Tiangen Biotech, Beijing, China), and $0.25 \mu \mathrm{M}$ SSR primers. Amplification parameters were as follows: 5 -min initial denaturation of the template DNA at $94{ }^{\circ} \mathrm{C} ; 35$ cycles of 45 -s template DNA denaturation at $94{ }^{\circ} \mathrm{C}$, 45-s primer annealing at the appropriate temperature, 45 -s primer extension at $72{ }^{\circ} \mathrm{C}$; and a final primer extension at $72{ }^{\circ} \mathrm{C}$ for 10 min. PCR products were stored at $4{ }^{\circ} \mathrm{C}$ before analysis by electrophoresis. SSR reactions were repeated for each SSR/DNA
Table 1. Species, sources, and characteristics of the 29 peach-related samples in Prunus.

\begin{tabular}{|c|c|c|c|}
\hline Code $^{z}$ & Prunus species & Source ${ }^{y}$ & Characteristic \\
\hline 1 & P. davidiana & NGRPC, Zhengzhou & Red flower \\
\hline 2 & P. davidiana & NGRPC, Zhengzhou & White flower \\
\hline 3 & P. davidiana & NGRPC, Nanjing & Red flower \\
\hline 4 & P. davidiana & NGRPC, Beijing & White flower \\
\hline 5 & P. kansuensis & NGRPC, Zhengzhou & Red root \\
\hline 6 & P. kansuensis & NGRPC, Zhengzhou & White root \\
\hline 7 & P. kansuensis & NGRPC, Nanjing & \\
\hline 8 & P. kansuensis & NGRPC, Beijing & Red root \\
\hline 9 & P. ferganensis & NGRPC, Zhengzhou & Kashi no.1 \\
\hline 10 & P. ferganensis & NGRPC, Zhengzhou & Kashi no.2 \\
\hline 11 & P. ferganensis & NGRPC, Nanjing & Datianren \\
\hline 12 & P. ferganensis & NGRPC, Beijing & \\
\hline 13 & P. mira & NGRPC, Zhengzhou & Linzhi, Tibet, China \\
\hline 14 & P. mira & NGRPC, Zhengzhou & Aba, Sichuan, China \\
\hline 15 & P. mira & NGRPC, Nanjing & \\
\hline 16 & P. mira & NGRPC, Beijing & \\
\hline 17 & P. persica & NGRPC, Beijing & Hubei, China \\
\hline 18 & P. persica & NGRPC, Beijing & Guizhou, China \\
\hline 19 & P. persica & NGRPC, Beijing & Sichuan, China \\
\hline 20 & P. persica & NGRPC, Beijing & Hebei, China \\
\hline 21 & unknown & ZFRI, CAAS & Chance seedling \\
\hline 22 & unknown & ZFRI, CAAS & Chance seedling \\
\hline 23 & unknown & ZFRI, CAAS & Chance seedling \\
\hline 24 & unknown & ZFRI, CAAS & Chance seedling \\
\hline 25 & unknown & ZFRI, CAAS & Chance seedling \\
\hline 26 & unknown & ZFRI, CAAS & Chance seedling \\
\hline 27 & unknown & ZFRI, CAAS & Chance seedling \\
\hline 28 & unknown & ZFRI, CAAS & Chance seedling \\
\hline 29 & P. persica & ZFRI, CAAS & Cultivar (Dajiubao) \\
\hline
\end{tabular}

${ }^{\mathrm{z}}$ Twenty-nine samples including 20 representative accessions of five species (codes 1-20) in Prunus, eight unknown samples awaiting species identification (codes 21-28), and one cultivar (code 29).

${ }^{y}$ National Germplasm Repository of Peach in China (NGRPC), Zhengzhou Fruit Research Institute (ZFRI), Chinese Academy of Agricultural Sciences CAAS).

sample combination at least twice to identify an appropriate annealing temperature and ensure consistent results.

DAta ANALYSIS. The 78 PCR products of 20 representative accessions were analyzed using electrophoresis with $9 \%$ polyacrylamide gels. Gels were silver stained according to the protocol described by Bassam et al. (1991). Optimal SSR primers for species identification were selected according to their consistent and reliable amplification patterns and interspecific discrimination power. Subsequent analysis was performed with fluorescent-labeled primers and CE. The selected SSR primers were labeled with fluorescein on the top of each forward primer ( $5^{\prime}$-FAM), and the PCR products of 29 samples were separated by $\mathrm{CE}$ and detected using a DNA analyzer (ABI 3730XL; Genewiz Biotechnology, Beijing, China). All the alleles of each locus were ranked in size from small to large, and a binary data matrix was established based on allele presence (1) or absence (0) at each locus for each sample. A dendrogram was constructed to represent the genetic relationships between the 29 individuals according to their similarity coefficients. The dendrogram was constructed using the unweighted pair group method with arithmetic means (UPGMA) in NTSYSpc software (version 2.10e; Applied Biostatistics, East Setauket, NY). 
Table 2. Simple sequence repeat (SSR) marker name and linkage group of the 78 SSRs used for peach related species identification. ${ }^{z}$

\begin{tabular}{|c|c|c|c|c|c|c|c|}
\hline SSR marker & Linkage group & SSR marker & Linkage group & SSR marker & Linkage group & SSR marker & Linkage group \\
\hline$\overline{\text { СРРСТ27 }}$ & 1 & UDP98-025 & 2 & ВРРСТ007 & 3 & pchgms2 & 4 \\
\hline СРРСТ26 & 1 & UDP96-013 & 2 & СРРСТ 18 & 3 & UDP96-003 & 4 \\
\hline СРРСТ29 & 1 & ВРРСТ034 & 2 & СРРСТ2 & 3 & ВРРСТ023 & 4 \\
\hline ВРРСТ028 & 1 & UDP98-406 & 2 & UDP96-008 & 3 & ВРРСТ031 & 4 \\
\hline ВРРСТ016 & 1 & ВРРСТ013 & 2 & ВРРСТ021 & 3 & ВРРСТ010 & 4 \\
\hline СРРСТ 16 & 1 & СРРСТ5 & 2 & СРDCT027 & 3 & СРРСТЗ & 4 \\
\hline UDP97-401 & 5 & СРРСТ8 & 6 & СРРСТ22 & 7 & ВРРСТ006 & 8 \\
\hline ВРРСТ017 & 5 & UDP96-001 & 6 & UDP98-405 & 7 & ВРРСТ019 & 8 \\
\hline ВРРСТ037 & 5 & ВРРСТ008 & 6 & UDP98-408 & 7 & UDP96-019 & 8 \\
\hline ВРРСТ038 & 5 & ВРРСТ025 & 6 & СРРСТ33 & 7 & СРРСТ6 & 8 \\
\hline ВРРСТ026 & 5 & СРРСТ 23 & 6 & СРSCT004 & 7 & MA013a & 8 \\
\hline pchgms4 & 5 & СРРСТ30 & 6 & СРSCT026 & 7 & MA017a & 8 \\
\hline
\end{tabular}

${ }^{\mathrm{z}}$ Ten representative loci were initially proposed in each Prunus linkage group based on the SSRs set proposed by Aranzana et al. (2003). A total of 78 SSRs were finally selected as candidates, as CPPCT5 and CPPCT3 occurred in two different linkage groups (highlighted in italics).

Table 3. The reasons for elimination of 60 simple sequence repeat (SSR) markers removed from further testing during prescreening on the 20 representative peach-related species accessions.

\begin{tabular}{|c|c|c|}
\hline Elimination reason & SSR marker & SSRs (no.) \\
\hline No amplification & СРРСТ17, СРРСТ18, СРРСТ29 & 3 \\
\hline Random amplification & ВРРСТ020, СРРСТ35, UDP96-003 & 3 \\
\hline Poor amplification & СРРСТ27, ВРРСТ038, UDP96-008 & 3 \\
\hline High intraspecific polymorphism & $\begin{array}{l}\text { ВРРСТ007, ВРРСТ008, ВРРСТ015, ВРРСТ030, ВРРСТ039, МА017a, UDP96-019, } \\
\text { UDP98-025, UDP98-409 }\end{array}$ & 9 \\
\hline Low interspecific polymorphism & 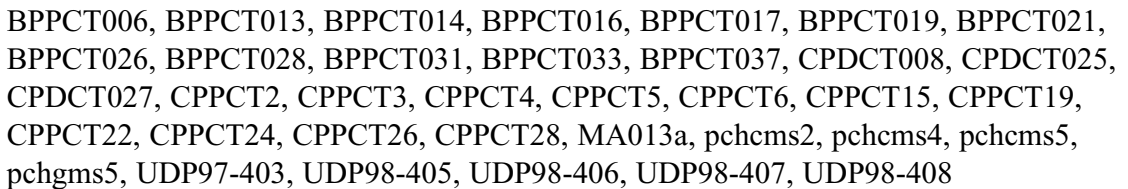 & 36 \\
\hline
\end{tabular}

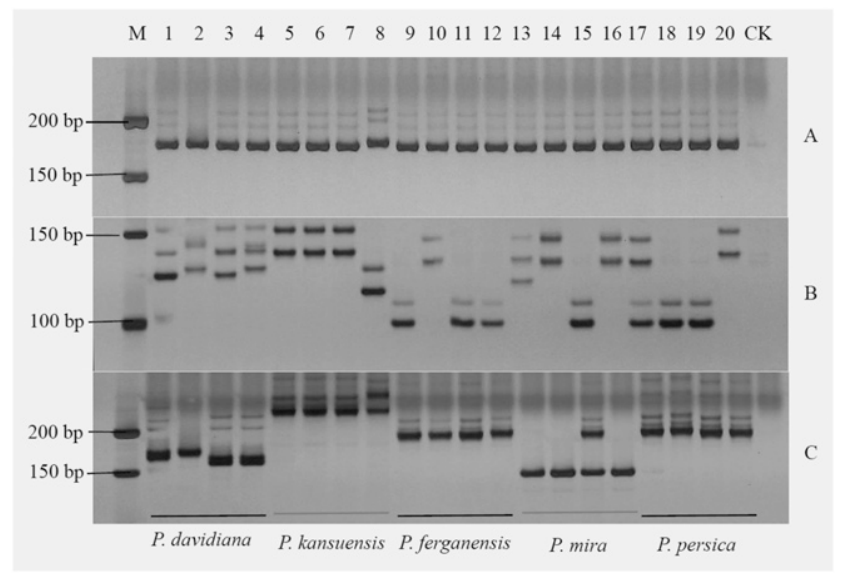

Fig. 1. Separation of amplification products generated by simple sequence repeat (SSR) primer (A) CPSCT026, (B) UDP98-025, and (C) CPPCT30 in 20 representative accessions in Prunus. $\mathrm{M}=\mathrm{DNA}$ marker, $\mathrm{CK}=$ blank control with double-distilled $\mathrm{H}_{2} \mathrm{O}$ replacing DNA template, codes $1-20=20$ representative accessions belonging to five species (P. davidiana, P. kansuensis, $P$. ferganensis, $P$. mira, and $P$. persica).

\section{Results}

SSR AMPLIFICATION AND SELECTION. The diversity of the 78 SSRs was assessed among the 20 representative peach samples of five species ( $P$. persica, $P$. davidiana, $P$. kansuensis, $P$. ferganensis, and $P$. mira) from NGRPC. Sixty loci were eliminated from further analysis for the following reasons (Table 3). Alleles could not be scored for nine primer pairs: three primer pairs failed to amplify fragments, three amplified more than two random fragments, and three loci were difficult to score due to poor amplification. Alleles at an additional 51 loci could be scored but were not sufficiently discriminatory. Of these, six loci were monomorphic for all 20 accessions in the electropherogram (Fig. 1A); 36 loci exhibited low interspecific polymorphism, usually due to some very high-frequency alleles; and nine loci exhibited high intraspecific polymorphism (Fig. 1B). Eighteen loci were selected for further testing. All of the 18 loci were consistently amplifiable, had only one or two alleles at a locus in every specific accession, and exhibited high interspecific polymorphism and low intraspecific polymorphism (Fig. 1C). 


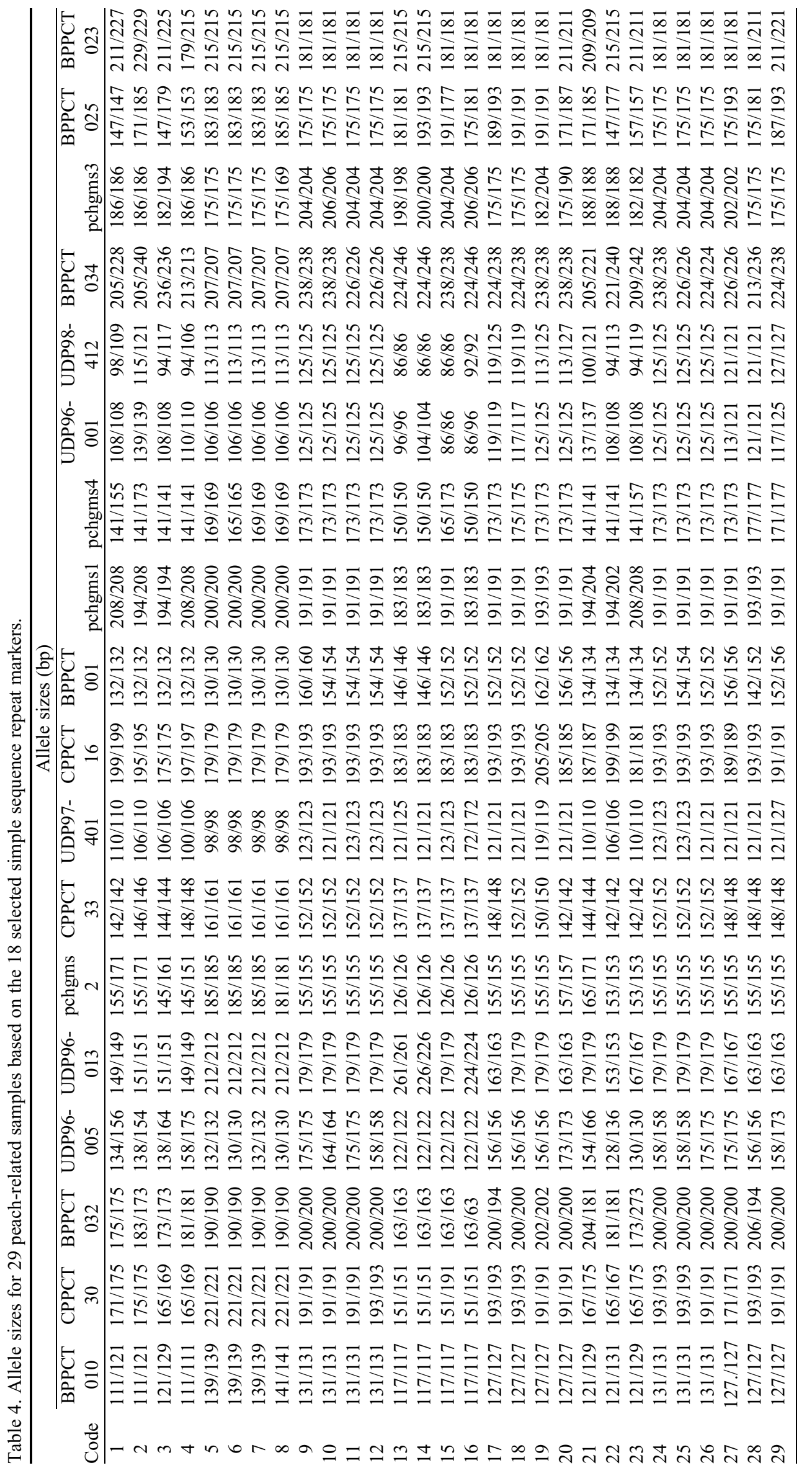


Table 5. Simple sequence repeat (SSR) genotype database for five peach-related species in Prunus based on 18 SSR markers and 20 accessions.

\begin{tabular}{|c|c|c|c|c|c|}
\hline \multirow[b]{2}{*}{ SSR marker } & \multicolumn{5}{|c|}{ Allele sizes (bp) } \\
\hline & P. davidiana & P. kansuensis & P. ferganensis & P. mira & P. persica \\
\hline ВРРСТ010 & $111 / 121 / 129$ & $139 / 141$ & 131 & 117 & 127 \\
\hline СРРСТ30 & $165 / 169 / 171 / 175$ & 221 & $191 / 193$ & $151 / 191$ & $191 / 193$ \\
\hline UDP96-005 & $134 / 138 / 154 / 156 / 158 / 164 / 175$ & $130 / 132$ & $158 / 164 / 175$ & 122 & $156 / 173$ \\
\hline UDP96-013 & $149 / 151$ & 212 & 179 & $179 / 224 / 226 / 261$ & $163 / 179$ \\
\hline pchgms2 & $145 / 151 / 155 / 161 / 171$ & $181 / 185$ & 155 & 126 & $155 / 157$ \\
\hline UDP97-401 & $100 / 106 / 110$ & 98 & $121 / 123$ & $121 / 123 / 125 / 172$ & $119 / 121$ \\
\hline СРРCТ16 & $175 / 195 / 197 / 199$ & 179 & 193 & 183 & $185 / 193 / 205$ \\
\hline ВРРСТ001 & 132 & 130 & $154 / 160$ & $146 / 152$ & $152 / 156 / 162$ \\
\hline pchgms1 & $194 / 208$ & 200 & 191 & $183 / 191$ & 191/193 \\
\hline pchgms4 & $141 / 155 / 173$ & $165 / 169$ & 173 & $150 / 165 / 173$ & $173 / 175$ \\
\hline UDP96-001 & $108 / 110 / 139$ & 106 & 125 & $86 / 96 / 104$ & $117 / 119 / 125$ \\
\hline ВРРСТ023 & $179 / 211 / 215 / 225 / 227 / 229$ & 215 & 181 & $181 / 215$ & $181 / 211$ \\
\hline
\end{tabular}

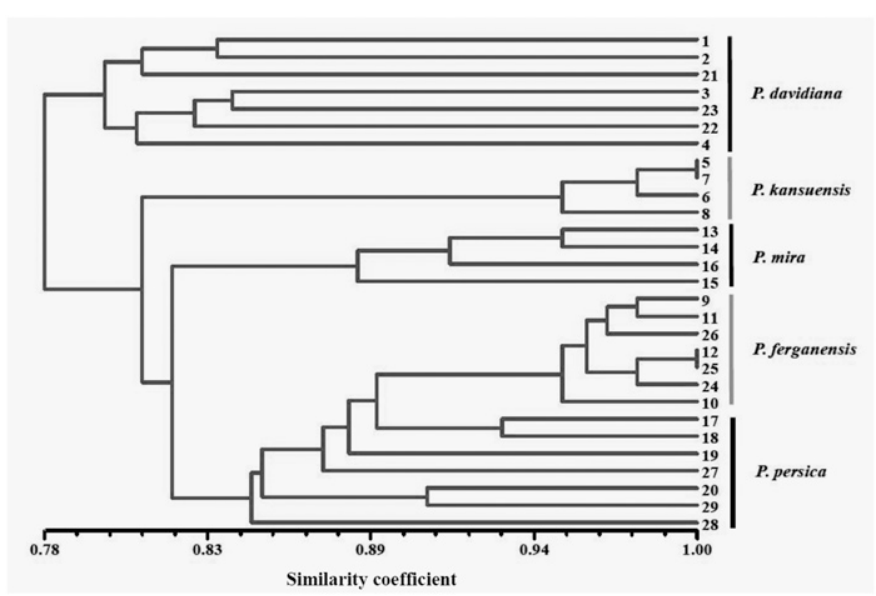

Fig. 2. Dendrogram of 29 accessions in Prunus resulting from the unweighted pair-group method with arithmetic means (UPGMA) cluster analysis based on the similarity coefficient obtained from simple sequence repeat (SSR) data. Twenty-nine accessions (codes 1-29) are divided into five clusters corresponding to five species ( $P$. davidiana, $P$. kansuensis, $P$. ferganensis, $P$. mira, and $P$. persica).

Development of a PEACH-Related SPECies GenOtype DATABASE USING FLUORESCENT-LABELED SSR MARKERS. The 18 SSR markers were used in fluorescent PCR and CE analysis, and a genotype database was generated for 20 representative accessions based on the 18 SSR markers (Table 4 ), then the genotype database for five species was generated based on the 18 SSR markers and 20 representative accessions (Table 5). The discrimination ability of 18 loci in the database varied and ranked from high (BPPCT010) to low (BPРCT023). The first five markers had high discrimination power and could be useful for the generation of a standard profile for species identification. Marker BPPCT010 can discriminate five species, and any marker of СРPCT30, BPPCT032, UDP96-005, and UDP96013 can be used to discriminate two species (Table 5). Adoption of the other markers for species identification would facilitate data sharing and help to correct for variation in data analysis between laboratories. The species $P$. kansuensis was the most easily identified among the five related species, and was one of the species that could be discriminated with most of the 18 selected SSR loci. The species $P$. mira, $P$. davidiana, $P$. ferganensis, and $P$. persica can also be discriminated by their unique loci (Table 5).

IDENTIFICATION OF UNKNOWN SAMPLE SPECIES. All the allele sizes of the eight unknown peach samples (codes 21-28) and 21 known accessions (codes 1-20, code 29) are presented in Table 4. A dendrogram of 29 samples was established (Fig. 2). Eight anonymous samples were included in three species clusters: samples 21, 22, and 23 were in the $P$. davidiana cluster; samples 24, 25, and 26 were in the $P$. ferganensis cluster; and samples 27 and 28 were in the $P$. persica cluster. As expected, sample 29, which was a $P$. persica cultivar, was found in the $P$. persica cluster. It was therefore possible to identify species by analysis with 18 SSR loci and phylogenetic comparison with the 20 known representative samples.

INTERSPECIFIC HYBRID ANALYSIS. Early identification of hybrids is desirable in peach cross breeding, and the selected 18 SSR markers can be used for hybrids analysis at the seedling stage. The $F_{1}$ hybrids share one allele with each of their parents on each locus, and progeny genotype(s) can therefore be predicted and proved by their parental genotypes. For example, only one genotype occurs in the $F_{1}$ hybrid on the locus of BPPCT010 in the hybridization of $P$. persica $\times P$. ferganensis (Fig. 3A), two genotypes on the locus of UDP96-005 in the hybridization of $P$. persica $\times P$. kansuensis (Fig. 3B), and four genotypes on the locus of UDP96-005 in the hybridization of $P$. persica $\times P$. davidiana (Fig. 3C).

\section{Discussion}

Peach cultivars can be easily distinguished using a unique DNA fingerprint (Li et al., 2013) or by molecular identity (Chen et al., 2011), because commercial peach clones are propagated by grafting. Peach-related species are wild resources in a mixed 


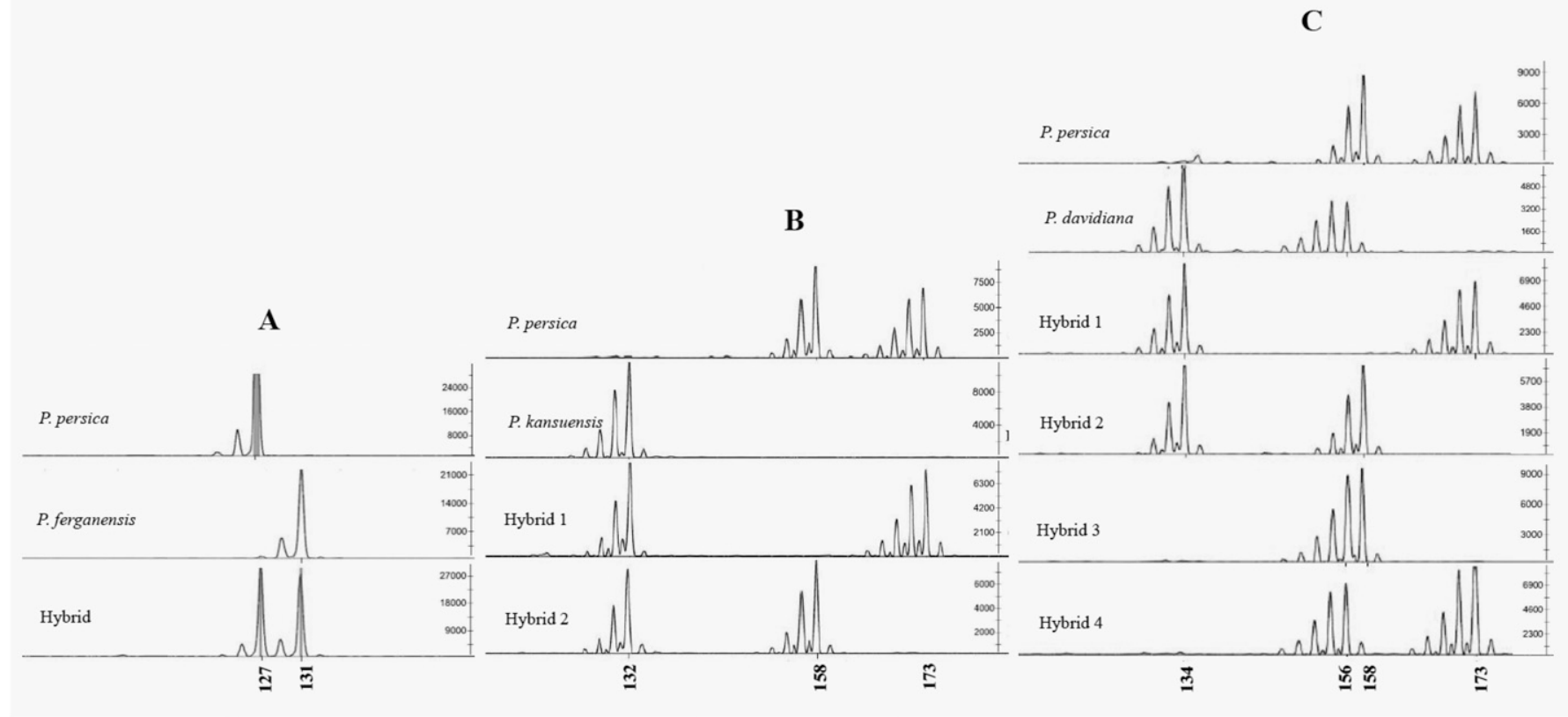

Fig. 3. ABI genotyper (Applied Biosystems, Foster City, CA) output of simple sequence repeat (SSR) profile of parents and progeny using for interspecific hybrid genotype analysis in Prunus. Allele sizes of the parents and their $\mathrm{F}_{1}$ hybrid(s) are indicated at each SSR loci: (A) profile for BPPCT010 of $P$. persica, $P$. ferganensis, and $\mathrm{F}_{1}$ hybrid ( $P$. persica $\times P$. ferganensis); $(\mathbf{B})$ profile for UDP96-005 of $P$. persica, $P$. kansuensis, and $\mathrm{F}_{1}$-hybrids $(P$. persica $\times P$. kansuensis); (C) profile for UDP96-005 of $P$. persica, $P$. davidiana, and $\mathrm{F}_{1}$-hybrids ( . persica $\times P$. davidiana). The $\mathrm{x}$ - and y-axes show allele size (in bps) and fluorescence intensity, respectively.

population that are commonly propagated by seed. We fully considered the sample origin, amount, and representativeness in this study. The 20 accessions used to screen for optimal SSRs all came from NGRPC, and different type and origin accessions were used in P. davidiana (red flower and white flower), P. kansuensis (red root and white root), P. mira (in Tibet and in Sichuan) and $P$. persica (in Huibei, Guizhou, Sichuan, and Hebei). Four accessions is a small sample; however, they were the recommended representative accessions from the three NGRPCs and should be considered as a starting point to which data from additional accessions and alleles can be added. Eight unknown accessions were tested for species identification, and three known interspecific hybrid populations were tested for hybrid analysis in this study.

Nine to ten representative loci were initially proposed in each Prunus linkage group; thus, a total of 78 SSRs were used as candidates. No more than 20 SSRs for validation were proposed prescreening with 20 representative accessions, resulting in 18 SSRs being finally selected on the basis of their amplification reliability and discrimination power. Some highly polymorphic SSRs from previous publications, such as BPPCT020 (Chen et al., 2011) and CPPCT22 (Bouhadida et al., 2011), were excluded from the current SSR panel. Use of loci with relatively few alleles simplified germplasm identification and increased efficiency of identification. Three known interspecific hybrids were used to evaluate transferability between species of the SSRs panel, and the results were in accord with their real identities.

A basic genotype database was established based on the 18 selected SSRs and 20 representative accessions, which should be useful for the identification of unknown Prunus accessions using dendrogram construction and cluster analysis. Fluorescent labeling and CE analyses improved the efficiency and accuracy of species identification in the current study. As allele size validation can be affected by primer tailing fluorescence chemicals, multiplexing PCR, and PCR product analysis (agarose gel, polyacrylamide gel, or capillary systems), SSR screening streamlining and database development for unknown samples identification and hybrid analysis using the same FAM-labeled forward primers, single-multiplexing PCR, and automatic CE analysis were used in this study. In fact, the relative range of allele sizes for five species on each locus is independent on the method used, which can be used as reference for species identification (Table 5). Eight unknown samples were successfully identified by the ZFRI, CAAS, which demonstrated the reliability of the genotype database and the SSRs panel.

Some peach-related species have disappeared from their original habitats in recent years due to climate change, overexploitation, pollution, and agricultural land reclamation (Xing et al., 2015; Zhong, 2008). On the other hand, accessions of species related to peach are preserved less frequently than those of cultivated peach, which are preserved at NGRPC. The practical genotyping developed in this study for five peach-related species will facilitate investigation and preservation of further wild accessions and allow the breeding potential of peach-related species to be fully realized. Peach-related species identification will be further enhanced by the availability of the peach genome sequence (Verde et al., 2013), the express sequence tag SSR database (Chen et al., 2014), microsatellite maps for the whole peach genome, and functional mining of genes associated with SSRs (Dettori et al., 2015; Zalapa et al., 2012).

\section{Conclusions}

In this study, 20 representative accessions at NGRPC were used to select for 18 SSRs, which were then successfully used for the efficient genotyping of five peach-related species 
including P. mira, P. davidiana, P. kansuensis, P. ferganensis, and $P$. persica. Based on the 18 SSRs and 20 representative accessions, a genotype database of the five species was established using fluorescent labeling and CE analysis. The SSR panel and genotype database were successfully used for the identification of eight unknown peach samples and three known interspecific hybrids analysis. The work presented provides a useful reference profile that should help facilitate peach germplasm management and peach breeding.

\section{Literature Cited}

Aranzana, M.J., J. Garcia-Mas, J. Carbó, and P. Arús. 2002. Development and variability analysis of microsatellite markers in peach. Plant Breed. 121:87-92.

Aranzana, M.J., A. Pineda, P. Cosson, E. Dirlewanger, J. Ascasibar, G. Cipriani, C.D. Ryder, R. Testolin, A. Abbott, G.J. King, A.F. Iezzoni, and P. Arús. 2003. A set of simple-sequence repeat (SSR) markers covering the Prunus genome. Theor. Appl. Genet. 106:819-825.

Bassam, B.J., G. Caetano-Anollés, and P.M. Gresshoff. 1991. Fast and sensitive silver staining of DNA in polyacrylamide gel. Anal. Biochem. 196:80-83.

Bouhadida, M., M.Á. Moreno, M.J. Gonzalo, J.M. Alonso, and Y. Gogorcena. 2011. Genetic variability of introduced and local Spanish peach cultivars determined by SSR markers. Tree Genet. Genomes 7:257-270.

Cao, K., L. Wang, G. Zhu, W. Fang, C. Chen, and P. Zhao. 2011. Construction of a linkage map and identification of resistance gene analog markers for root-knot nematodes in wild peach, Prunus kansuensis. J. Amer. Soc. Hort. Sci. 136:190-197.

Cao, K., Z. Zheng, L. Wang, X. Liu, G. Zhu, W. Fang, S. Cheng, P. Zeng, C. Chen, X. Wang, M. Xie, X. Zhong, X. Wang, P. Zhao, C. Bian, Y. Zhu, J. Zhang, G. Ma, C. Chen, Y. Li, F. Hao, Y. Li, G. Huang, Y. Li, H. Li, J. Guo, X. Xu, and J. Wang. 2014. Comparative population genomics reveals the domestication history of the peach, Prunus persica, and human influences on perennial fruit crops. Genome Biol. 15:415.

Chen, C., K. Cao, L. Wang, G. Zhu, and W. Fang. 2011. Molecular ID establishment of main China peach varieties and peach related species. Zhongguo Nong Ye Ke Xue 44:2081-2093.

Chen, C., C.H. Bock, W.R. Okie, F.G. Gmitter, Jr., S. Jung, D. Main, T.G. Beckman, and B.W. Wood. 2014. Genome-wide characterization and selection of expressed sequence tag simple sequence repeat primers for optimized marker distribution and reliability in peach. Tree Genet. Genomes 10:1271-1279.

Cheng, F.S., S.K. Brown, and N.F. Weeden. 1997. A DNA extraction protocol from various tissues in woody species. HortScience 32:921922.

Cheng, Z., Z. Chen, C. Hu, X. Deng, and Z. Luo. 2001. The identification and systematic relationship analysis of Amygdalus spp. by molecular markers (in Chinese). J. Huazhong Agr. Univ. 20:199-204.

Cheng, Z. and H. Huang. 2009. SSR fingerprinting Chinese peach cultivars and landraces (Prunus persica) and analysis of their genetic relationships. Sci. Hort. 120:188-193.

Dangl, G.S., J. Yang, D.A. Golino, and T. Gradziel. 2009. A practical method for almond cultivar identification and parental analysis using simple sequence repeat markers. Euphytica 168:41-48.

Decroocq, V., M.G. Favé, L. Hagen, L. Bordenave, and S. Decroocq. 2003. Development and transferability of apricot and grape EST microsatellite markers across taxa. Theor. Appl. Genet. 106:912-922. Dettori, M.T., S. Micali, J. Giovinazzi, S. Scalabrin, I. Verde, and G. Cripriani. 2015. Mining microsatellites in the peach genome: Development of new long-core SSR markers for genetic analyses in five Prunus species. Springerplus 4:337.

Dirlewanger, E., P. Cosson, M. Tavaud, M.J. Aranzana, C. Poizat, A. Zanetto, P. Arús, and F. Laigret. 2002. Development of microsatellite markers in peach [Prunus persica (L.) Batsch] and their use in genetic diversity analysis in peach and sweet cherry (Prunus avium L.). Theor. Appl. Genet. 105:127-138.

Emanuelli, F., S. Lorenzi, L. Grzeskowiak, V. Catalano, M. Stefanini, M. Troggio, S. Myles, J.M. Martinez-Zapater, E. Zyprian, F.M. Moreira, and M.S. Grando. 2013. Genetic diversity and population structure assessed by SSR and SNP markers in a large germplasm collection of grape. BMC Plant Biol. 13:39.

Guichoux, E., L. Lagache, S. Wagner, P. Chaumeil, P. Léger, O. Lepais, C. Lepoittevin, T. Malausa, E. Revardel, F. Salin, and R.J. Petit. 2011. Current trends in microsatellite genotyping. Mol. Ecol. Resour. 11:591-611.

Guo, Z., H. Ge, X. Wang, and J. Yan. 1996. The analysis of chromosome karyotypes and taxonomic relationships of Amygdalus plants. Yuan Yi Xue Bao 23:223-226.

Kalia, R.K., M.K. Rai, S. Kalia, R. Singh, and A.K. Dhawan. 2011. Microsatellite markers: An overview of the recent progress in plants. Euphytica 177:309-334.

Lacis, G., I. Rashal, S. Ruisa, V. Trajkovski, and A.F. Iezzoni. 2009. Assessment of genetic diversity of Latvian and Swedish sweet cherry (Prunus avium L.) genetic resources collections by using SSR (microsatellite) markers. Sci. Hort. 121:451-457.

Lambert, P. and T. Pascal. 2011. Mapping Rm2 gene conferring resistance to the green peach aphid (Myzus persicae Sulzer) in the peach cultivar "Rubira ${ }^{\circledR}$ ". Tree Genet. Genomes 7:1057-1068.

Layne, D.R. and D. Bassi. 2008. The peach: Botany, production and uses. CAB Intl., Wallingford, UK.

Li, T., Y. Li, Z. Li, H. Zhang, Y. Qi, and T. Wang. 2008. Simple sequence repeat analysis of genetic diversity in primary core collection of peach (Prunus persica). J. Integr. Plant Biol. 50:102-110.

Li, X., X. Meng, H. Jia, L. Wang, Z. Wang, R. Ma, D. Wu, B. Dong, M.J. Aranzana, P. Arús, and Z. Gao. 2013. Construction of peach genotype database with fluorescent-labeled SSR markers (in Chinese). Guoshu Xuebao 30:924-932.

Liu, X., G. Reighard, G.A. Swire-Clark, W.C. Bridges, A.G. Abbott, and W.V. Baird. 2009. Identification of the chromosomal genomic regions associated with peach tree short life syndrome using microsatellite/ SSR markers. HortScience 44:1175-1176.

Ma, L., D. Kong, H. Liu, S. Wang, Y. Li, and X. Pang. 2012. Construction of SSR fingerprint on 36 Chinese jujube cultivars. Yuan Yi Xue Bao 39:647-654.

Mnejja, M., J. Garcia-mas, W. Howad, and P. Arús. 2005. Development and transportability across Prunus species of 42 polymorphic almond microsatellites. Mol. Ecol. Notes 5:531-535.

Mnejja, M., J. Garcia-mas, W. Howad, M.L. Badenes, and P. Arús. 2004. Simple-sequence repeat (SSR) markers of japanese plum (Prunus salicina Lindl.) are highly polymorphic and transferable to peach and almond. Mol. Ecol. Notes 4:163-166.

Moing, A., J. Pöessel, L. Svanella-Dumas, M. Loonis, and J. Kervella. 2003. Biochemical basis of low fruit quality of Prunus davidiana, a pest and disease donor for peach breeding. J. Amer. Soc. Hort. Sci. 128:55-62.

Mowrey, B.D., D.J. Werner, and D.H. Byrne. 1990. Isozyme survey of various species of Prunus in the subgenus Amygdalus. Sci. Hort. 44:251-260.

Sosinski, B., M. Gannavarapu, L.D. Hager, L.E. Beck, G.J. King, C.D. Ryder, S. Rajapakse, W.V. Baird, R.E. Ballard, and A.G. Abbott. 2000. Characterization of microsatellite markers in peach [Prunus persica (L.) Batsch]. Theor. Appl. Genet. 101:421-428.

Testolin, R., T. Marrazo, G. Cipriani, R. Quarta, I. Verde, M.T. Dettori, M. Pancaldi, and S. Sansavini. 2000. Microsatellite DNA in peach (Prunus persica L. Batsch) and its use in fingerprinting and testing the genetic origin of cultivars. Genome 43:512-520.

Verde, I., A.G. Abbott, S. Scalabrin, S. Jung, S. Shu, F. Marroni, T. Zhebentyayeva, M.T. Dettori, J. Grimwood, F. Cattonaro, A. Zuccolo, L. Rossini, J. Jenkins, E. Vendramin, L.A. Meisel, V. Decroocq, B. Sosinski, S. Prochnik, T. Mitros, A. Policriti, G. Cipriani, L. Dondini, S. Ficklin, D.M. Goodstein, P. Xuan, C.D. Fabbro, V. Aramini, D. Copetti, S. Gonzalea, D.S. Horner, R. Falchi, S. Lucas, E. Mica, J. Maldonado, B. Lazzari, D. Bielenberg, R. Pirona, 
M. Miculan, A. Barakat, R. Testolin, A. Stella, S. Tartarini, P. Tonutti, P. Arús, A. Orellana, C. Wells, D. Main, G. Vizzotto, H. Silva, F. Salamini, J. Schmutz, M. Morgante, and D.S. Rokhsar. 2013. The high-quality draft genome of peach (Prunus persica) identifies unique patterns of genetic diversity, domestication and genome evolution. Nat. Genet. 45:487-494.

Verde, I., N. Bassil, S. Scalabrin, B. Gilmore, C.T. Lawley, K. Gasic, D. Micheletti, U.R. Rosyara, F. Cattonaro, E. Vendramin, D. Main, V. Aramini, A.L. Blas, T.C. Mockler, D.W. Bryant, L. Wilhelm, M. Troggio, B. Sosinski, M.J. Aranzana, P. Arús, A. Iezzoni, M. Morgante, and C. Peace. 2012. Development and evaluation of a 9K SNP array for peach by internationally coordinated SNP detection and validation in breeding germplasm. PLoS One 7:e35668. Wang, Z. and J. Zhou. 1990. Pollen morphology of peach germplasm. Yuan Yi Xue Bao 17:161-168.

Wang, Z. and E. Zhuang. 2001. China fruit monograph: Peach flora. China Forestry Press, Beijing, China.
Xing, C., Y. Tian, F. Guan, and F. Meng. 2015. Evaluation of genetic diversity in Amygdalus mira (Koehne) Ricker using SSR and ISSR markers. Plant Syst. Evol. 301:1055-1064.

Yamamoto, T., K. Mochida, T. Imai, Y.Z. Shi, I. Ogiwara, and T. Hayashi. 2002. Microsatellite markers in peach [Prunus persica (L.) Batsch] derived from an enriched genomic and cDNA libraries. Mol. Ecol. Notes 2:298-301.

$\mathrm{Yu}$, M., R. Ma, J. Xu, Z. Shen, and Z. Zhang. 2004. Identification of genetic relationship of peach species by SSR (in Chinese). Guoshu Xuebao 21:106-112.

Zalapa, J.E., H. Cuevas, H. Zhu, S. Steffan, D. Senalik, E. Zeldin, B. McCown, R. Harbut, and P. Simon. 2012. Using next-generation sequencing approches to isolate simple sequence repeat (SSR) loci in the plant science. Amer. J. Bot. 99:193-208.

Zhong, Z. 2008. Studies on resource ecology of Prunus mira Koehne (Amygdalus mira Koehne kow et. Kpst) in Xizang (Tibet) Linzhi. MS Thesis, Univ. Tibet, Linzhi, China. 